\title{
Outcome determinants of urethroplasty in the management of inflammatory anterior urethral strictures
}

\author{
F M Claassen, ${ }^{1}$ MMed (Urol), PhD (Urol); S B A Mutambirwa, ${ }^{2}$ MMed (Urol); L Potgieter, ${ }^{3} \mathrm{PhD}(\mathrm{Pharmacol}) ; \mathrm{L} \mathrm{Botes,}{ }^{4} \mathrm{PhD}$; \\ H F Kotze, ${ }^{3} \mathrm{PhD}$ (Haematol), DSc; F E Smit, ${ }^{3}$ MMed (Cardiothorac Surg), $\mathrm{PhD}$ \\ ${ }^{1}$ Department of Urology, Faculty of Health Sciences, University of the Free State, Bloemfontein, South Africa \\ ${ }^{2}$ Department of Urology, Sefako Makgatho Health Sciences University, and Dr George Mukhari Academic Hospital, Pretoria, South Africa \\ ${ }^{3}$ Department of Cardiothoracic Surgery, Faculty of Health Sciences, University of the Free State, Bloemfontein, South Africa \\ ${ }^{4}$ Department of Health Sciences, Faculty of Health and Environmental Sciences, Central University of Technology, Bloemfontein, South Africa
}

Corresponding author: F M Claassen (claassen@ufs.ac.za)

\begin{abstract}
Background. Limited data are available on outcomes of the surgical management of inflammatory urethral strictures secondary to infection, a major cause of stricture. Several shortcomings that need to be addressed have been identified in the past.

Objective. To determine the impact of stricture length, position and degree of obliterative urethral lumen on the surgical outcomes of corrective procedures for inflammatory anterior urethral strictures.

Methods. This retrospective analysis used the records of patients who presented with proven infective anterior urethral strictures at an academic hospital from 2007 to 2010. All patients were followed up after 48 months. Urethroplasty outcomes were analysed according to stricture location and length and effect of urethral obliteration.

Results. The median age of the 174 patients in the study was 47 (range 21 - 86) years. Anastomotic urethroplasty was successful in $59 / 99$ (59.6\%) patients. Augmented anastomotic urethroplasty was successful in 11/15 (73.3\%) patients. Dorsal onlay buccal mucosa graft urethroplasty was successful in 23/32 (71.9\%) patients, significantly higher than in $2 / 9$ (22.2\%) patients who underwent ventral onlay buccal mucosa graft urethroplasty ( $p=0.017$; hazard ratio $3.4 ; 95 \%$ confidence interval $1.29-9.40)$. The one-stage circular pedicled penile skin-flap urethroplasty was successful in $1 / 12(8.3 \%)$ patients. Two-stage urethroplasty was successful in 5/7 (71.4\%) patients. A primary component analysis of the 73 failed procedures showed that stricture length was the main contributor to failure (eigenvalue 1.79; 45\%).

Conclusions. Urethroplasty remains a challenge in inflammatory urethral strictures, where stricture length was the main reason for treatment failure.
\end{abstract}

S Afr Med J 2019;109(12):947-951. https://doi.org/10.7196/SAMJ.2019.v109i12.14003

There is a paucity of published data addressing outcomes of the surgical management of inflammatory urethral strictures secondary to infection, the main cause of stricture in the developing world. ${ }^{[1,2]}$ The aetiology of stricture in the developed world was analysed by Lumen et al. ${ }^{[3]}$ who demonstrated that the main contributing causes were instrumentation (32\%), idiopathic conditions (23\%), urethral catheters (13\%), trauma (3\%) and infection (3\%).

Limited literature is available on inflammatory urethral strictures secondary to Neisseria gonorrhoeae (gonococcal) infection. In available studies, several shortcomings were identified. Firstly, analyses and comparison of data on outcomes are complicated by the non-uniform nature of urethral strictures that differ in length, location and aetiology. ${ }^{[4]}$ Secondly, studies do not distinguish between inflammatory and traumatic aetiology. Finally, infective urethral strictures have been identified as a risk factor for urethroplasty failure.$^{[5]}$ As the inflammatory process differs significantly from other causes of urethral obstruction and stricture, published surgical data on the selection and outcomes of procedures performed in a noninflammatory setting might not be applicable in an inflammatory setting. This can explain the high failure rate in the management of inflammatory stricture. ${ }^{[5]}$

The aim of this study was to determine the outcomes of different surgical procedures in inflammatory urethral stricture in relation to stricture length, position and degree of obliteration of the urethral lumen by analysing the success rates after the first interventions performed at an academic hospital in central South Africa (SA).

\section{Methods}

A retrospective, analytical cohort study of 174 consecutive patients who underwent surgical treatment for inflammatory urethral strictures between 2007 and 2010 was performed using hospital records. Patients with a history of purulent urethral discharge and urethral stricture disease were included. Patients with a history of trauma, iatrogenic, catheter-related strictures and Lichen sclerosis were excluded. Patients were included in this study after a minimum of 48 months' follow-up, which was determined from the day of treatment to the last clinic follow-up date.

Outcomes of different surgical procedures for inflammatory urethral strictures were analysed in three categories: $(i)$ in relation to the length of the stricture; (ii) in relation to the position of the stricture; and (iii) whether the stricture was obliterative or not. The length of the strictures (in centimetres) was determined by retrograde and prograde urethrograms. The location of the lesions was documented as penile, penobulbar, bulbar and panurethral. Pan-urethral stricture disease was identified if the patient had low-grade strictures throughout the length of the penile and bulbar urethra. Lesions were classified as obliterative or nonobliterative, based on the preoperative retrograde urethrogram (RUG). Functional success was assigned if the patient reported an improvement in voiding symptoms and urinary quality of life. When a patient presented with obstructive lower-urinary tract symptoms suggestive of recurrence, a follow-up RUG was performed. Treatment failure was defined as recurrence of symptoms requiring 
reinvestigation (RUG) or any intervention for stricture recurrence. Data were analysed to determine the role of procedure type, length, position and obliterative urethral lumens on outcomes and surgical management of inflammatory urethral strictures.

\section{Statistical analysis}

The Kaplan-Meier estimator was used to calculate failure-specific stricture-free rates. Stricture-free rates were measured in months and censored on the date that a patient developed stricture recurrence. The frequencies were subjected to a $\chi^{2}$ test. Statistical significance was set at $p<0.05$. The effect size was calculated using Cramer's V statistic. This test statistic is used to measure the strength of association between two nominal variables, the values ranging between 0 and 1 . Values close to 0 indicate a weak association and those close to 1 indicate a strong association between the variables. Analyses were done using SPSS version 15.0 (SPSS Inc, USA). A primary component analysis (PCA) was performed on the variables in the failed procedures to determine the variables that contributed the most to failure, and included stricture length, position and presenting symptoms (R software).

\section{Ethical approval}

Ethial approval to conduct the study was obtained from the Ethics Committee, University of the Free State, Bloemfontein, SA (ref. no. ECUFS 124/2015) and relevant managerial members of staff.

\section{Results}

\section{Stricture characteristics and procedures}

A total of 174 patients (median age 47 (range 21 - 86) years) with infective urethral strictures underwent treatment for anterior urethral stricture from 2007 to 2010 . The location of the urethral strictures was as follows: penile ( $n=17 ; 9.8 \%)$, penobulbar $(n=61 ; 35.1 \%)$, bulbar $(n=68 ; 39.1 \%)$ and pan-urethral $(n=28 ; 16.1 \%)$. Patients presented with the following symptoms: acute urinary retention secondary to obliterated urethral lumens $(n=113 ; 64.9 \%)$, obstructive lower-urinary tract symptoms with non-obliterated lumens $(n=45$; $25.9 \%)$ and perineal sepsis $(n=16 ; 9.2 \%)$. The median follow-up was 63 (range 48 - 70) months. The surgical outcomes per procedure are presented in Table 1. The overall success rate was 58.0\% (101/174).

Dorsal onlay buccal mucosa graft urethroplasty (DBMGU) was successful in 23/32 (71.8\%) patients, which was significantly higher than in $2 / 9(22.2 \%)$ patients who underwent ventral onlay buccal mucosa graft urethroplasty (VBMGU) $(p=0.017$; hazard ratio (HR) 3.4; $95 \%$ confidence interval (CI) 1.29 - 9.40). One-stage circular pedicled penile skin-flap urethroplasty (cPFU) was successful in 1/12 (8.3\%) patients, and two-stage urethroplasty was successful in 5/7 (71\%) patients.

\section{Procedures and stricture length}

The success rate of procedures in relation to stricture length is summarised in Table 2. Anastomotic urethroplasty (AR), anastomotic urethroplasty augmented with buccal mucosa (AAR) and DBMGU had success rates of $>65 \%$. For stricture lengths of $2.1-4.0 \mathrm{~cm}$, the most successful outcomes were achieved with DBMGU $(81.3 \%)$ and AAR (75.0\%). Two-stage urethroplasty, where buccal mucosal graft (BMG) was combined with ventral pedicled penile skin flap, achieved a $71 \%$ success rate for strictures $>4 \mathrm{~cm}$.

\section{Stricture location}

As shown in Table 3, AR and AAR were successful in patients with strictures in the penobulbar area (65.8\% and $71.4 \%$, respectively). AAR was successful in $80.0 \%$ of patients with strictures in the bulbar

\begin{tabular}{lllll}
\multicolumn{6}{l}{ Table 1. Surgical outcomes analyses of $\mathbf{1 7 4}$ patients treated for inflammatory urethral stricture } \\
\hline Procedure & Patients, $\boldsymbol{n}$ & Age, mean (range), years & Success rate, $\boldsymbol{n}$ (\%) & Follow-up, mean (range), months \\
\hline AR & 99 & $45.9(21-80)$ & $59(59.6)$ & $59.5(48-70)$ \\
AAR & 15 & $43.2(30-76)$ & $11(73.3)$ & $58(48-65)$ \\
DBMGU & 32 & $52.5(21-82)$ & $23(71.9)$ & $58.3(48-65)$ \\
VBMGU & 9 & $47.8(25-73)$ & $2(22.2)$ & $59(50-62)$ \\
cPFU & 12 & $52.4(35-72)$ & $1(8.3)$ & $60(48-66)$ \\
Two-stage urethroplasty & 7 & $45.8(34-68)$ & $5(71.4)$ & $56(48-58)$
\end{tabular}

$\mathrm{AR}=$ anastomotic urethroplasty; $\mathrm{AAR}=$ anastomotic urethroplasty augmented with buccal mucosa; DBMGU = dorsal onlay buccal mucosa graft urethroplasty; VBMGU = ventral onlay buccal mucosa graft urethroplasty; cPFU = circular pedicled penile skin-flap urethroplasty.

Table 2. Outcomes for specific stricture length groups, excluding perineum urethrostomies and Johanson procedures

\begin{tabular}{|c|c|c|c|c|c|c|}
\hline Procedure & Length, cm & Length, mean (range), $\mathrm{cm}$ & Patients, $n$ & Success rate, $n(\%)$ & $p$-value & CI for effect size $w$ \\
\hline \multirow[t]{2}{*}{$\mathrm{AR}$} & $0.1-2.0$ & $1.3(0.3-7.0)$ & 82 & $53(64.6)$ & 0.122 & $\mathrm{w}=0.158$ \\
\hline & $2.1-4.0$ & & 14 & $6(42.9)$ & & $95 \%$ CI $0.00-0.36$ \\
\hline \multirow[t]{2}{*}{ AAR } & $0.1-2.0$ & $3.1(1.0-6.0)$ & 4 & $4(100)$ & 0.273 & $\mathrm{w}=0.316$ \\
\hline & $2.1-4.0$ & & 8 & $6(75.0)$ & & $95 \%$ CI $0.00-0.88$ \\
\hline \multirow[t]{2}{*}{ DBMGU } & $0.1-2.0$ & $4.6(0.6-10.0)$ & 7 & $5(71.4)$ & 0.599 & $\mathrm{w}=0.110$ \\
\hline & $2.1-4.0$ & & 16 & $13(81.3)$ & & $95 \%$ CI $0.00-0.51$ \\
\hline \multirow[t]{2}{*}{ VBMGU } & $0.1-2.0$ & $3.5(1.0-4.5)$ & 2 & $1(50.0)$ & 0.346 & $\mathrm{w}=0.333$ \\
\hline & $2.1-4.0$ & & 6 & $1(16.7)$ & & $95 \%$ CI $0.00-1.03$ \\
\hline \multirow[t]{2}{*}{$\mathrm{cPFU}$} & $0.1-2.0$ & $5.0(3.7-7.0)$ & 0 & $0(0)$ & Not estimable & Not estimable \\
\hline & $2.1-4.0$ & & 4 & $0(0)$ & & \\
\hline Two-stage & $0.1-2.0$ & $8.0(4.0-9.0)$ & 0 & $0(0)$ & - & Sample size too small \\
\hline urethroplasty & $2.1-4.0$ & & 1 & $1(100)$ & & \\
\hline
\end{tabular}

$\mathrm{CI}=$ confidence interval; $\mathrm{AR}$ = anastomotic urethroplasty; $\mathrm{AAR}=$ anastomotic urethroplasty augmented with buccal mucosa; DBMGU = dorsal onlay buccal mucosa graft urethroplasty; $\mathrm{CI}=$ confidence interval; $\mathrm{AR}=$ anastomotic urethroplasty; $\mathrm{AAR}=$ anastomotic urethroplasty augmented with buccal
$\mathrm{VBMGU}=$ ventral onlay buccal mucosa graft urethroplasty; $\mathrm{CPFU}=$ circular pedicled penile skin-flap urethroplasty. 
region. DBMGU achieved a success rate of $76.9 \%$ in the penile region, with favourable outcomes in the penobulbar (66.7\%) and bulbar (80.0\%) regions. Information of patients with pan-urethral strictures are not included in Table 3.

\section{Outcomes of procedures in relation to obliterative v. non- obliterative lumens}

AR, AAR and DBMGU had success rates of $64 \%, 73 \%$ and $67 \%$, respectively, in patients with obliterative lumens (Table 4).Two-stage urethroplasty BMG combined with pedicled penile skin flap had a higher success rate than one-stage $\mathrm{CPFU}$ in patients with obliterative lumens, i.e. $71 \%$ and $83 \%$, respectively $(p=0.010)$.

PCA was performed on the 73 patients in whom procedures had failed to determine which of the variables (stricture length, position and presenting symptoms) contributed significantly to outcome. In the 73 patients in whom the first procedures had failed, stricture length contributed $45 \%$ and position $27 \%$ to variance of the data. The eigenvalues of these two variables were $>1$, indicating that the variables were the primary components that caused failure. Stricture length - not position - was the main contributor in failed urethroplasty: AR (49\%), DBMGU (74\%), VBMGU (59\%) and one-stage cPFU (62\%).

\section{Discussion}

In this study, the long-term outcomes of surgical interventions in inflammatory strictures were evaluated. An attempt was made to relate outcomes of the specific procedure to the length and location of the stricture. The impact on the outcome of whether the stricture was obliterative, was also considered.

The overall success rate for endoscopic and urethroplasty procedures was $50 \%$. This finding could be attributed to the postinfective inflammatory process and underestimation of stricture length with $\mathrm{RUG}^{[5]}$ when used as the only imaging modality. ${ }^{[6]}$ In patients with infective urethral strictures, urethral ultrasound examination in addition to RUG determines the stricture length more accurately, and preoperative ultrasonography has been recommended to accurately assess the extent of spongiofibrosis in an effort to decrease the recurrence rate. ${ }^{[7,8]}$ However, due to anatomical limitations, ultrasonography is not recommended as the sole assessment tool for strictures and should be combined with urethrography. The use of ultrasonography changed the surgical approach in 19\% of patients in a retrospective study that compared ultrasonography and RUG. ${ }^{[7]}$ The best results in our series were obtained when stricture repair was augmented with a dorsal onlay BMG in both AAR and DBMGU.

\begin{tabular}{|c|c|c|c|c|c|}
\hline Procedure & Stricture position & Patients, $n$ & Success rate, $n(\%)$ & $p$-value & CI for effect size w \\
\hline \multirow[t]{3}{*}{$\mathrm{AR}$} & Penile & 1 & $0(0)$ & 0.400 & $\mathrm{w}=0.141$ \\
\hline & Penobulbar & 38 & $25(65.8)$ & & $95 \%$ CI $0.00-0.32$ \\
\hline & Bulbar & 53 & $34(64.2)$ & & \\
\hline \multirow[t]{3}{*}{ AAR } & Penile & 2 & $2(100)$ & 0.308 & $\mathrm{w}=0.490$ \\
\hline & Penobulbar & 7 & $5(71.4)$ & & $95 \%$ CI $0.00-0.89$ \\
\hline & Bulbar & 5 & $4(80.0)$ & & \\
\hline \multirow[t]{3}{*}{ DBMGU } & Penile & 13 & $10(76.9)$ & 0.811 & $\mathrm{w}=0.173$ \\
\hline & Penobulbar & 12 & $8(66.7)$ & & $95 \%$ CI $0.00-0.39$ \\
\hline & Bulbar & 5 & $4(80.0)$ & & \\
\hline \multirow[t]{3}{*}{ VBMGU } & Penile & 1 & $1(100)$ & 0.076 & $\mathrm{w}=0.756$ \\
\hline & Penobulbar & 3 & $1(33.3)$ & & $95 \%$ CI $0.00-16.50$ \\
\hline & Bulbar & 5 & $0(0)$ & & \\
\hline \multirow[t]{3}{*}{$\mathrm{cPFU}$} & Penile & 2 & $1(50.0)$ & 0.141 & $\mathrm{w}=0.674$ \\
\hline & Penobulbar & 8 & $0(0)$ & & $95 \%$ CI $0.00-1.14$ \\
\hline & Bulbar & 1 & $0(0)$ & & \\
\hline
\end{tabular}

Table 4. Procedure success rates in non-obliterative and obliterative lumens and perineal sepsis

\begin{tabular}{|c|c|c|c|c|c|c|c|c|}
\hline \multirow[b]{2}{*}{ Procedure } & \multicolumn{2}{|c|}{$\begin{array}{c}\begin{array}{c}\text { Non-obliterative } \\
\text { lumens }\end{array} \\
\end{array}$} & \multicolumn{2}{|c|}{ Obliterative lumens } & \multicolumn{2}{|c|}{ Perineal sepsis } & \multirow[b]{2}{*}{$p$-value } & \multirow[b]{2}{*}{ CI for effect size w } \\
\hline & $n$ & Successful, $n(\%)$ & $n$ & Successful, $n(\%)$ & $n$ & Successful, $n(\%)$ & & \\
\hline $\mathrm{AR}$ & 17 & $8(47)$ & 78 & $50(64)$ & 4 & $1(25)$ & 0.153 & $\begin{array}{l}\mathrm{w}=0.195 \\
95 \% \text { CI } 0.00-13.76\end{array}$ \\
\hline AAR & 2 & $1(50)$ & 11 & $8(77)$ & 2 & $1(50)$ & 0.525 & $\begin{array}{l}\mathrm{w}=0.213 \\
95 \% \text { CI } 0.00-5.76\end{array}$ \\
\hline \multicolumn{9}{|l|}{ Substitution urethroplasty } \\
\hline DBMGU & 1 & $1(100)$ & 27 & $18(67)$ & 4 & $4(100)$ & 0.314 & $\begin{array}{l}\mathrm{w}=0.269 \\
95 \% \text { CI } 0.00-10.59\end{array}$ \\
\hline VBMGU & $\mathrm{n} / \mathrm{a}$ & $\mathrm{n} / \mathrm{a}$ & 9 & $2(22)$ & $\mathrm{n} / \mathrm{a}$ & $\mathrm{n} / \mathrm{a}$ & $\mathrm{n} / \mathrm{a}$ & $\mathrm{n} / \mathrm{a}$ \\
\hline cPFU & $\mathrm{n} / \mathrm{a}$ & $\mathrm{n} / \mathrm{a}$ & 10 & $1(10)$ & 2 & $0(0)$ & 0.640 & $\begin{array}{l}\mathrm{w}=0.250 \\
95 \% \text { CI } 0.00-5.76\end{array}$ \\
\hline Two-stage urethroplasty & $\mathrm{n} / \mathrm{a}$ & $\mathrm{n} / \mathrm{a}$ & 1 & $1(10)$ & 6 & $5(83)$ & $\mathrm{n} / \mathrm{a}$ & $\mathrm{n} / \mathrm{a}$ \\
\hline
\end{tabular}


These procedures possibly compensated for the incorrect diagnosis of stricture length.

Stricture length remained the main determinator of success of the procedures. No difference in outcome of AR for lengths $<2 \mathrm{~cm}$ or $\geq 2 \mathrm{~cm}$ in predominantly non-inflammatory strictures was reported. ${ }^{\left[{ }^{[]}\right.}$ Eltahawy et al.$^{[9]}$ reported a $99 \%$ success rate in 260 patients with mainly trauma- and catheter-related urethral strictures (mean length $1.9 \mathrm{~cm}$ ) located in the bulbar urethra. ${ }^{[9]} \mathrm{A}$ trauma-related bulbar stricture length of $2 \mathrm{~cm}$ is considered ideal to treat by means of AR, with high success rates having been reported. ${ }^{[10]}$ However, Morey and Kizer ${ }^{[11]}$ reported a $91 \%$ success rate in 11 patients with bulbar urethral strictures of $2.6-5.0 \mathrm{~cm}$, who underwent AR. Although AR is reserved for stricture lengths $<2 \mathrm{~cm}$, Aghaji and Odoemene ${ }^{[12]}$ reported a success rate of $88 \%$ in their group of 98 patients, where the stricture length was $3 \mathrm{~cm}$. With regard to inflammatory strictures reported in our study, a much lower success rate of $65 \%$ with AR was obtained in patients with bulbar strictures $<2 \mathrm{~cm}$ and a relatively low success rate of $43 \%$ for strictures $2-4 \mathrm{~cm}$. AR also had a significantly better outcome in strictures $<2 \mathrm{~cm}$ than in those $>2 \mathrm{~cm}$. It is therefore apparent from this study that in inflammatory urethral strictures, AR should only be considered for shorter stricture lengths and definitely in cases of $<2 \mathrm{~cm}$.

The AAR technique, which combines the anastomotic urethroplasty with substitution graft, has been suggested for bulbar strictures of $2-4 \mathrm{~cm} \cdot{ }^{[13]}$ Hoy et al. ${ }^{[14]}$ reported a $96.9 \%$ patency rate with a dorsal onlay AAR for a mean stricture length of up to $4.6 \mathrm{~cm} .{ }^{[14]}$ In our study, AAR achieved a success rate of $73 \%$ in 15 patients, which was markedly lower than the $90 \%$ reported previously. ${ }^{[15]}$ A notable finding was that AR had a lower success rate (65\%) than AAR (100\%) or DBMGU (71\%), even for stricture lengths of $0.1-2.0 \mathrm{~cm}$. This finding once again raises the question of underestimation of stricture length and suggests that AAR and DBMGU procedures compensate for this by means of RUG.

The preferred approach of many institutions for bulbar strictures $>4 \mathrm{~cm}$ is circumferential urethral reconstruction with a patch or vascularised flap. Whitson et al. ${ }^{[16]}$ reported a 10-year stricture recurrence-free rate of 79\% in patients who underwent cPFU. However, in our study, contradictory results were found in inflammatory strictures, where $\mathrm{CPFU}$ had an overall success rate of only $8 \%$ - much lower than the success rates of DBMGU (72\%) and two-stage urethroplasty (71\%).

Strictures $>4 \mathrm{~cm}$ were identified as an important risk factor for failure. The preferred approach is a circumferential repair with skin flaps or grafts. ${ }^{[5,17]}$ The best results for strictures $>4 \mathrm{~cm}$ were obtained with DBMGU (56\%) and two-stage urethroplasty (80\%).

In this study, infective strictures were located mainly in the bulbar and penobulbar urethra, contrary to the penile urethra reported in the literature. ${ }^{[18]}$ Stricture location has an effect on stricture length - penile strictures tend to be longer than bulbar strictures, with mean stricture lengths of $6.1 \mathrm{~cm}$ and $3.1 \mathrm{~cm}$, respectively. ${ }^{[18]}$ We found that stricture location did not affect treatment outcome. However, penile strictures require more complex procedures owing to the thinner spongiosum in the penile urethra compared with the bulbar urethra. ${ }^{[4]}$ In patients who underwent urethroplasty, the stricture location had no negative effect on treatment outcome. This is in contrast to Breyer et al.'s ${ }^{[2]}$ findings, where strictures located in the penile urethra had a higher recurrence rate after repair.

In this study, obliterative urethral lumens had no negative influence on success rates for AR, AAR and dorsal BMG urethroplasties. In summary, obliterative urethral lumens played no role in the complexity of urethral strictures when urethroplasty was done, mainly because the obliterative lumens were excised or augmented to a favourable calibre. This is one of the reasons why obliterative urethral lumens were excluded from the urethral score validation. ${ }^{[4]}$

\section{Study limitations}

The retrospective nature of this study and because procedures were performed by different surgeons, complicated data interpretation. The selection of procedures was biased because it was done by different surgeons with different skill levels.

\section{Conclusions}

Underestimation of inflammatory urethral stricture length was the major single contributor towards failed procedures. In general, more invasive procedures yielded better results in inflammatory strictures than published outcomes of procedures in non-inflammatory lesions. Therefore, AR can be considered for lesions $<2 \mathrm{~cm}$, AAR for lesions $2-3 \mathrm{~cm}$ and DBMGU for lesions $2-4 \mathrm{~cm}$ in length. Two-stage urethroplasty is preferable to treat patients with stricture lengths $>4 \mathrm{~cm}$. Location, and to a lesser extent symptoms, played a minor role in failures. In patients with infective urethral strictures the placement of the BMG may determine the success of substitution urethroplasty. The BMG must be placed dorsally instead of ventrally. Obliterative urethral lumens did not have a negative effect on the success rate of urethroplasty in patients with infective strictures.

Declaration. This article is based on research conducted by FMC in partial fulfilment of his $\mathrm{PhD}$ thesis.

Acknowledgements. Dr Daleen Struwig, medical writer/editor, for technical and editorial preparation of the manuscript.

Author contributions. FMC: formulated the research aims and objectives, collected the data and prepared the first draft of the manuscript; SBAM: assisted with the second draft of the manuscript; LP: performed the statistical analysis and interpreted the data; LB: prepared the first draft of the manuscript and drew the tables; HFK: prepared the first draft of the manuscript; FES: finalised and approved the final version of the manuscript.

Funding. None.

Conflicts of interest. None.

1. Heyns CF van der Merwe J, Basson J, van der Merwe A. Treatment of male urethral strictures possible reasons for the use of repeated dilatation or internal urethrotomy rather than urethroplasty. Afr J Surg 2012;50(3):82-87.

2. Breyer BN, McAninch JW, Whitson JM, et al. Multivariate analysis of risk factors for long-term urethroplasty outcome. J Urol 2010;183(2):613-617. https://doi.org/10.1016/j.juro.2009.10.018

3. Lumen N, Hoebeke P, Willemsen P, de Troyer B, Pieters R, Oosterlinck W. Etiology of urethral stricture disease in the 21st century. J Urol 2009;182(3):983-987. https://doi.org/10.1016/j.juro.2009.05.023

4. Eswara JR, Han J, Raup T, et al. Refinement and validation of the urethral stricture score in categorizing anterior urethral stricture complexity. Urology 2015;85(2):474-477.

. Mathur RK, Nagar M, Mathur R, Khan F, Deshmukh C, Guru N. Single-stage preputial skin island flap urethroplasty for long-segment urethral strictures: Evaluation and determinants of success. Br J Urol Int 2014;113(1):120-126. https://doi.org/10.1111/bju.12361

6. El-Ghar MA, Osman Y, Elbaz E, Refiae H, El-Diasty T. MR urethrogram versus combined retrograde rethrogram and sonourethrography in diagnosis of urethral stricture. Eur J Radiol 2010:74(3):193-198.

Barbagli G, Palminteri E, Lazzeri M, Guazzoni G. Anterior urethral strictures. Br J Urol Int 2003;92(5):497-505

8. Choudhary S, Singh P, Sundar E, Kumar S, Sahai A. A comparison of sonourethrography and retrograde urethrography in the evaluation of anterior urethral strictures. Clin Radiol 2004;59(8):736-742. https://doi.org/10.1016/j.crad.2004.01.014

9. Eltahawy EA, Virasoro R, Schlossberg SM, McCammon KA, Joran GH. Long-term follow-up for excision and primary anastomosis for anterior urethral strictures. J Urol 2007;177(5):1803-1806.

10. Santucci RA, Mario LA, McAninch JW. Anastomotic urethroplasty for bulbar urethral strictures: Analysis of 168 patients. J Urol 2002;167(4):1715-1719. https://doi.org/10.1016/S0022-5347(05)65184-1

1. Morey AF, Kizer WS. Proximal bulbar urethroplasty via extended anastomotic approach - what are the limits? J Urol 2006;175(6):2145-2149. https://doi.org/10.1016/S0022-5347(06)00259-X

12. Aghaji $\mathrm{AE}$, Odoemene CA. One-stage urethroplasty for strictures: Nigerian experience. Int J Urol 2001;8(7):380-385. https://doi.org/10.1046/j.1442-2042.2001.00317.x

13. Guralnick ML, Webster GD. The augmented anastomotic urethroplasty: Indications and outcome in 29 patients. J Urol 2001;165(5):1496-1501. https://doi.org/10.1016/S0022- 5347(05)66335-5

14. Hoy NY, Kinnaird A, Rourke KF. Expanded use of a dorsal onlay augmented anastomotic urethroplasty with buccal mucosa for long segment bulbar urethral strictures: Analysis of outcomes and complications. Urology 2013;81(6):1357-1361. https://doi.org/10.1016/j.urology.2013.02.012 
15. Abouassaly R, Angermeier A. Augmented anastomotic urethroplasty. J Urol 2007;177(6):2211-2216. https://doi.org/10.1016/j.juro.2007.01.140

16. Whitson JM, McAninch JW, Elliott SP, Alsikafi NF. Long-term efficacy of distal penile circular fasciocutaneous flaps for single stage reconstruction of complex anterior urethral stricture disease. J Urol 2008;179(6):2259-2264. https://doi.org/10.1016/j.juro.2008.01.087

17. Carney KJ, McAninch JW. Penile circular fasciocutaneous flaps to reconstruct complex anterior urethral strictures. Urol Clin North Am 2002;29(2):397-409. https://doi.org/10.1016/S00940143(02)00046-0
18. Fenton AS, Morey AF, Aviles R, Garcia CR. Anterior urethral strictures: Etiology and characteristics. Urology 2005;65(6):1055-1058. https://doi.org/10.1016/j.urology.2004.12.018 